\title{
A Switched-Capacitor degenerated, scalable $g m-C$ filter-bank for acoustic front-ends
}

\author{
Komail Badami \\ KU Leuven - ESAT - MICAS, \\ Belgium \\ komail.badami@esat.kuleuven.be
}

\author{
V. Rajesh Pamula \\ IMEC, \\ Belgium \\ pamula@imec.be
}

\author{
Marian Verhelst \\ KU Leuven - ESAT - MICAS \\ Belgium \\ marian.verhelst@kuleuven.be
}

\begin{abstract}
Filter-banks based on a $g m-C$ topology are popular in acoustic sensor systems targeting spectral analysis. Their benefits lie in a very low power consumption and center-frequency scalability through $g m$-tuning to cover the audio frequency range. However the linear signal swing at the output of the filter is limited due to the inherent non-linearity of the input transistors in a differential pair. This work assesses the impact of noise and center-frequency specifications on the power consumption of 2 OTA based $g m-C$ bandpass filters, both from a theoretical and practical point of view. Next, we introduce a novel scalable switched-capacitor based degeneration technique that enhances the linear signal swing at the filter output. Simulation results in 90nm CMOS demonstrate a power consumption of only $44 \mathrm{nW}$ for a bandpass filter with Q-factor of 1 with $63 \mathrm{~dB}$ dynamic range $(<2 \%$ THD) and a center-frequency of $100 \mathrm{~Hz}$. This scales to only $1.4 \mu \mathrm{W}$ for a center-frequency at $3.2 \mathrm{kHz}$. These power consumption numbers compare favorably with the state-of-theart and enhance the Figure of Merit by more than $1.5 \mathrm{X}$ for a similar dynamic range.
\end{abstract}

\section{INTRODUCTION}

Acoustic processing for power constrained applications such as cochlear-implants or voice activity detection systems, often decompose the audio signal into multiple frequency bins for time-spectral analysis. Such analysis helps to identify patterns in the acoustic spectrum for tasks such as recognition or classification [1], [2]. Analog bandpass filter-banks based on a $g m-C$ topology are commonly used for these applications and typically cover the acoustic frequency range from few tens of $\mathrm{Hz}$ to a few $\mathrm{kHz}$. For a low input referred noise, it is desirable to precede these filter-banks by a high gain amplifier and hence these filters need to handle large signal swings without introducing significant distortion. Existing filter-banks use non-standard CMOS transistors such as floating gate devices [1], [3] or require potentials larger than $V_{D D}$ [4] to achieve linearity and $g m$ based center-frequency $\left(f_{c}\right)$ scalability. The commonly used resistor based degeneration technique to improve linearity is not suitable in the acoustic frequency range, as very large resistors $(\sim 100 M \Omega)$ are needed that incur area penalty for implementation in a CMOS process.

This work theoretically assesses the noise and frequency dependent bounds on the power-consumption of bandpass $g m-C$ filters in Section II, after which Section III introduces a novel switched-capacitor based degeneration technique that enhances the linear signal swing at the filter output and moreover allows for convenient $f_{c}$ scaling in the filter-banks.

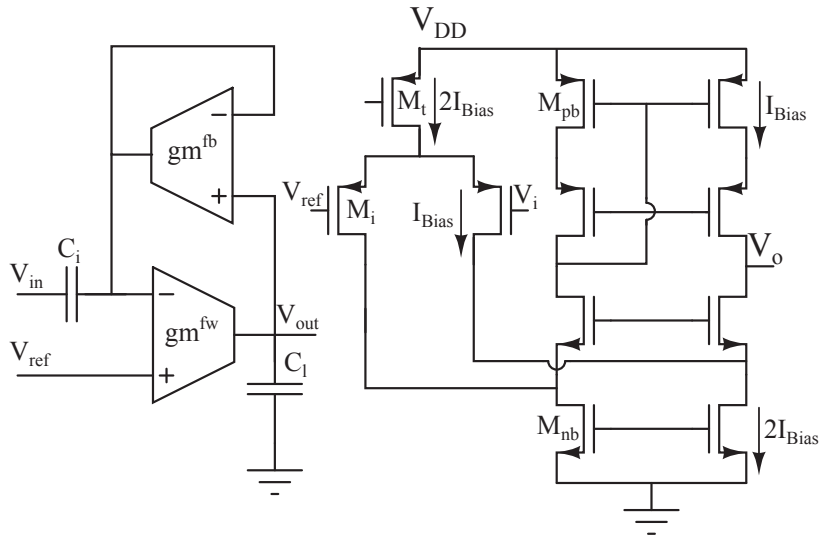

Fig. 1. $g m-C$ filter architecture used in analysis (left) and the folded cascode transconductor (right) used for $g m$ cell implementation

\section{THEORETICAL ANALYSIS FOR POWER BOUNDS ON $g m-C$ FILTERS}

$g m-C$ based filter architectures are the most commonly used topology in acoustic filter-bank applications, as they allow very low power operation together with frequency tunability, albeit with a limited linear swing at the transconductor output. Switched-capacitor filters, on the other hand, allow for a wider linear output swing, however at a much larger power consumption than $g m-C$ filters. This power penalty stems from the need for a unity gain bandwidth well beyond the used switching clock frequency. Detailed analysis for frequency and noise specification aware power bound for a 2 OTA based bandpass $g m-C$ filters is presented in this section.

The $g m-C$ filter architecture analyzed for noise and frequency dependent power bound estimation is shown in Fig.1. Although this analysis assumes a folded cascode implementation for the transconductor as shown in Fig. 1, right, it can easily be adapted to other transconductor topologies. The transfer function of BPF in Fig.1 can be written as,

$$
\frac{v_{\text {out }}}{v_{\text {in }}}=\frac{-s \frac{C_{\text {in }}}{g m_{i}^{f b}}}{1+s \frac{C_{l}}{g m_{i}^{f w}}+s^{2} \frac{C_{l} C_{\text {in }}}{g m_{i}^{f b} g m_{i}^{f w}}}
$$

where the superscripts $f b$ and $f w$ indicate contributions from the feedback and the forward path respectively. Also, the subscript text in the $g m$ corresponds to the transistor naming in the transconductor cell. Comparing the denominator of Eq. 1 


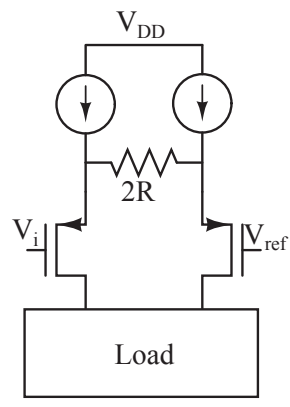

(a)

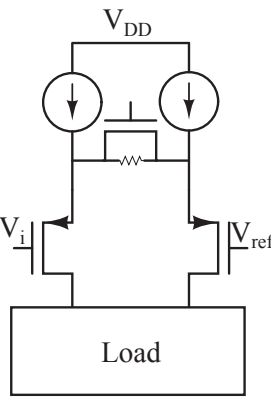

(b)

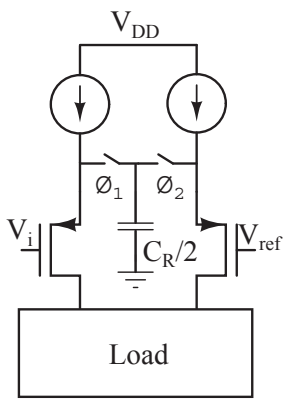

(c)
Fig. 2. Traditional resistive (a) and MOSFET in triode (b) based degeneration. Proposed switched-capacitor (c) based degenernation

with the standard second order denominator polynomial shows that the $f_{c}$ and $\mathrm{Q}$ factor are defined by

$$
f_{c}=\frac{1}{2 \pi} \sqrt{\frac{g m_{i}^{f b}}{C_{i n}} \frac{g m_{i}^{f w}}{C_{l}}} \quad Q=\sqrt{\frac{g m_{i}^{f w}}{g m_{i}^{f b}} \frac{C_{i n}}{C_{l}}}
$$

The noise current spectral density at the filter output due to noise generated in the forward path can be written as

$$
S_{I o}^{f w}=\frac{16}{3} k T\left(g m_{i}^{f w}+g m_{n b}^{f w}+g m_{p b}^{f w}\right)
$$

The transistor sizes are assumed to be sufficiently large so that the flicker noise contribution is limited to less than $10 \%$ of the total noise contribution and is ignored in the further analysis. The cascode transistors are biased such that their noise contribution is insignificant. Similarly the noise current spectral density at output due to the feedback path can be written as

$$
S_{I o}^{f b}=\frac{16}{3} k T\left(g m_{i}^{f b}+g m_{n b}^{f b}+g m_{p b}^{f b}\right)\left(Z i n(s) g m_{i}^{f w}\right)^{2}
$$

where $\operatorname{Zin}(s) \approx \frac{1}{s C i n} \| g m_{i}^{f b}$ is the impedance looking into the inverting node of the forward path. From Eq. 3 and 4 the total noise current density at the output node is

$$
S_{I o}^{\text {total }}=S_{I o}^{f w}+S_{I o}^{f b}
$$

Without loss of generality, further analysis assumes that the same transconductor is used in the forward and the feedback path i.e $g m_{x}^{f w}=g m_{x}^{f b}=g m_{x}$. This allows the total noise current spectral density at the filter output to be expressed as

$$
\begin{aligned}
S_{I o}^{\text {total }}=\frac{16}{3} k T\left(g m_{i}+g m_{n b}+\right. & \left.g m_{p b}\right) \\
& \left(1+\left(\operatorname{Zin}(s) g m_{i}\right)^{2}\right)
\end{aligned}
$$

For frequencies around $f_{c},|Z i n(s)| \approx \frac{1}{2 g m_{i}}$ hence the total integrated output noise power over $f_{ \pm 3 d B}$ can be expressed as

$$
\begin{aligned}
v_{n o}^{2} & \approx \frac{20}{3} k T \frac{\left(g m_{i}+g m_{n b}+g m_{p b}\right)}{2 \pi} \int_{f_{-3 d B}}^{f_{3 d B}}\left(\frac{1}{f C_{l}}\right)^{2} d f \\
& \approx \frac{20}{3}\left(\frac{k T}{C_{l}}\right) \frac{g m_{i}+g m_{n b}+g m_{p b}}{2 \pi C_{l}} \frac{f_{3 d B}-f_{-3 d B}}{f_{3 d B} f_{-3 d B}}
\end{aligned}
$$

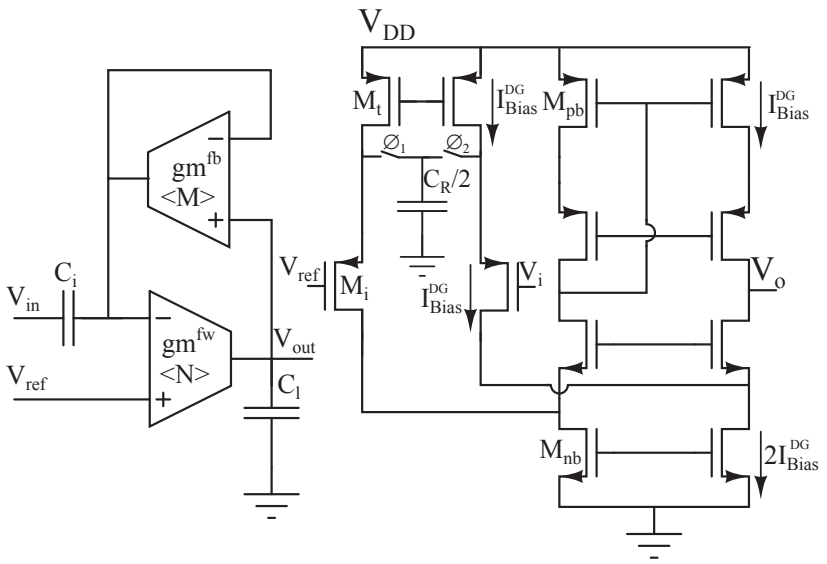

Fig. 3. Switched-capacitor degenerated $g m-C$ filter (left) and the degenerated transconductor implementation (right). $M$ and $N$ indicate the number of parallel cells in the feedback and the forward path respectively

Furthermore, as the bias current through $M_{i}$ and $M_{p b}$ are identical and about one half of current through $M_{n b}, g m_{i}+$ $g m_{n b}+g m_{p b}$ will typically range between $3 g m_{i}$ to $4 g m_{i}$. Also, Eq. 8 allows to approximate input referred noise $v_{n i} \approx$ $v_{n o}$ in the frequency range of $f_{ \pm 3 d B}$ as in this frequency range $\left|v_{\text {out }} / v_{\text {in }}\right| \approx 1$. Although this ignores the second order transfer function and omits integration beyond $f_{ \pm 3 d B}$ range, simulation results in Section IV across the whole $0.1 f_{-3 d B}$ to $10 f_{3 d B}$ range will demonstrate a discrepancy of only $\sim 30 \%$ due to this approximation. Power bounds can be computed by assuming a $g m / I_{\text {bias }}=\alpha$ with Eq. 8

$$
\begin{aligned}
v_{n o}^{2} \approx \frac{20}{3}\left(\frac{k T}{C_{l}}\right) \frac{I_{\text {bias }}\left(\alpha_{i}+2 \alpha_{n b}+\alpha_{p b}\right)}{2 \pi C_{l}} & \left(\frac{f_{3 d B}-f_{-3 d B}}{f_{3 d B} f_{-3 d B}}\right)
\end{aligned}
$$

The output linear range of the filter topology in Fig. 1 is limited by the linearity of the input transistor pair of the forward and the feedback path. Due to unity gain feedback path, the signal swing at the inverting node of the forward path equals the swing of the output signal $v_{\text {out }}$. The maximum swing that the input pair can tolerate is limited to $\sqrt{2}\left(V_{G S}-V_{T}\right)$ for a hard non-linearity. For a $90 \mathrm{~nm}$ CMOS technology, and a dc-bias point of $V_{D D} / 2=600 \mathrm{mV}$, the maximum signal swing would be limited to less than $100 m V p p$ for similar $V_{G S}-V_{T}$ for transistors $M_{i}$ and $M_{t}$. Hence even though $g m-C$ filters are attractive from the power consumption point of view, the output linear swing is quite limited. The next Section will therefore introduce a novel switched-capacitor based degeneration technique which exactly enhances the linear signal swing at the output of $g m-C$ filters and is also perfectly suitable for standard CMOS implementation.

\section{SWITCHED CAPACITOR BASED DEGENERATION}

Resistive degeneration as shown in Fig. 2(a) allows local feedback and reduces the signal swing across the input tran- 
TABLE I

TARGET SPECIFICATIONS

\begin{tabular}{|c|c|c|c|c|}
\hline $\begin{array}{c}\text { num. of } \\
\text { channels }\end{array}$ & $f_{c}$ range $(\mathrm{Hz})$ & Q factor & $\begin{array}{c}\text { Integrated } \\
\text { output noise }\end{array}$ & $\begin{array}{c}\text { Output signal } \\
\text { swing }\end{array}$ \\
\hline 6 & $\begin{array}{c}100-3200 \\
\text { Octave spacing }\end{array}$ & 1 & $<100 \mu V_{r m s}$ & $\begin{array}{c}>400 m V p p \\
<2 \% \text { THD }\end{array}$ \\
\hline
\end{tabular}

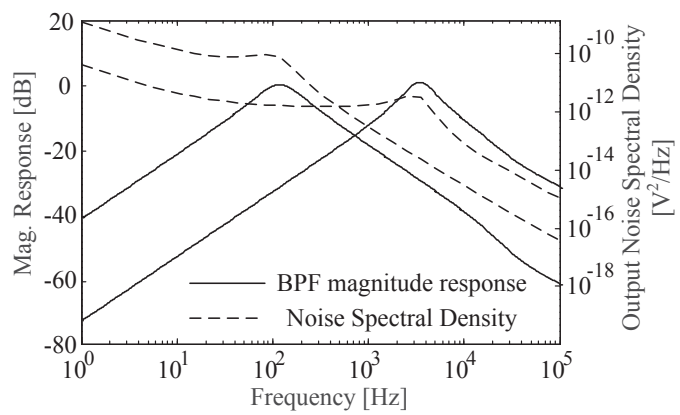

Fig. 4. Simulated magnitude response and output noise voltage spectral density $S_{v o}(f)$ for the switched-capacitor degenerated filter for the first and the last channel in $90 \mathrm{~nm}$ CMOS

sistors. This allows a wider linear swing at the output. The effective transconductance of a degenerated differential pair is

$$
g m_{e f f}=\frac{g m_{i}}{1+g m_{i} R} \approx \frac{1}{R} \quad \text { if } \quad g m_{i} R>>1
$$

For the audio-frequency range, the necessary resistor value for $g m_{i} R>>1$ tends to be of the order of $\sim 100 M \Omega$ for capacitor value of few tens of $\mathrm{pF}$. Such a resistor is uncomfortably large to be implemented as a resistor in a CMOS process. Moreover, the resistance value is not very well controlled in CMOS processes hence restricting the use for such applications. Degeneration may be also achieved by using transistors in triode region as shown in Fig. 2(b). However, they are of limited use because for larger signal swings, the degenerating transistors are no longer in deep triode region and the value of the induced resistor would vary with the signal amplitude which defeats its purpose.

This work proposes a switched-capacitor based degeneration, as shown in Fig. 2(c). The effective resistance between the two source nodes in Fig. 2(c) can be expressed as $2 /\left(f_{s} C_{R}\right)$. We will demonstrate that such an implementation is extremely linear and allows for $f_{c}$ and Q-factor scalable filter design by varying the number of parallel $g_{m}$ cells in the feedback and forward path. Moreover, also at run-time $f_{c}$ can be fine tuned by varying the $f_{s}$ or switched-capacitor, $C_{R}$, or a combination of both. The effective transconductance can be then expressed from Eq. 10 as

$$
g m_{\text {eff }} \approx f_{s} C_{R} \quad \text { if }\left(g m_{i} / f_{s} C_{R}\right)>>1
$$

The resulting filter topology with the same switched capacitor based degeneration in the forward and the feedback path is shown in Fig. 3. The transfer function for this filter for one $g m$ cell in the forward and the feedback path $(M=N=1)$ can be expressed as

$$
\frac{v_{\text {out }}}{v_{\text {in }}} \approx \frac{-s \frac{C_{i n}}{f_{s} C_{R}}}{1+s \frac{C_{l}}{f_{s} C_{R}}+s^{2} \frac{C_{l} C_{i n}}{f_{s}^{2} C_{R}^{2}}}
$$

TABLE II

SiMULATED NOISE AND LINEARITY RESULTS IN 90 $\mathrm{nm}$ CMOS.

\begin{tabular}{|c|c|c|}
\hline$f_{c}(\mathrm{~Hz})$ & Integrated output noise & Output signal swing \\
\hline \multirow{3}{*}{100} & $87 \mu V_{r m s}$ & \\
& $f_{-3 d B}<f<f_{+3 d B}$ & $510 m V_{p p} ;<1 \%$ THD \\
& $121 \mu V_{r m s}$ & $800 m V_{p p} ;<5 \%$ THD \\
& $0.1 f_{-3 d B}<f<10 f_{+3 d B}$ & \\
\hline \multirow{3}{*}{3200} & $86 \mu V_{r m s}$ & \\
& $f_{-3 d B}<f<f_{+3 d B}$ & $484 m V_{p p} ;<2 \%$ THD \\
& $116 \mu V_{r m s}$ & $700 m V_{p p} ;<5 \%$ THD \\
& $0.1 f_{-3 d B}<f<10 f_{+3 d B}$ & \\
\hline
\end{tabular}

This allows the $f_{c}$ and $\mathrm{Q}$ factor to be defined by a tunable capacitor ratio, being

$$
f_{c} \approx \frac{1}{2 \pi} \sqrt{\frac{g m_{\text {eff }}^{2}}{C_{l} C_{\text {in }}}} \approx \frac{f_{s}}{2 \pi} \sqrt{\frac{C_{R}}{C_{i n}} \frac{C_{R}}{C_{l}}} \quad Q=\sqrt{\frac{C_{i n}}{C_{l}}}
$$

By similar analysis as in Section II, the total output noise current density $S_{I o}^{f w}$ due to the forward path can be written as

$$
S_{I o}^{f w}=\frac{16}{3} k T\left(g m_{t}^{f w}+g m_{n b}^{f w}+g m_{p b}^{f w}\right)+8 k T f_{s} C_{R}
$$

The noise contribution of the input transistors in the degenerated transconductor is not significant, as they effectively appear as cascode devices. Due to splitting of the tail current sources, their noise contribution is no longer common mode and contributes to output noise as in Eq. 14. The last term of Eq. 14 is the noise contribution of the switched-capacitor based degeneration resistor. Following a similar analysis as in Section II the total output noise can hence be estimated as

$$
\begin{array}{r}
v_{n o}^{2} \approx \frac{k T}{C_{l}}\left(\frac{20}{3} \frac{g m_{t}+g m_{n b}+g m_{p b}}{2 \pi C_{l}}+10 \frac{f_{s} C_{R}}{2 \pi C_{l}}\right) \\
\left(\frac{f_{3 d B}-f_{-3 d B}}{f_{3 d B} f_{-3 d B}}\right)
\end{array}
$$

Similar to Eq. 9, the power consumption can be estimated from $I_{b i a s}^{D G}$ from the equation below

$$
v_{n o}^{2} \approx \frac{k T}{C_{l}}\left(\frac{\overbrace{\frac{20 \frac{I_{\text {bias }}^{D G}\left(\alpha_{t}+2 \alpha_{n b}+\alpha_{p b}\right)}{2 \pi C_{l}}}{\text { dominant }}+\overbrace{10 \frac{f_{s} C_{R}}{2 \pi C_{l}}}^{\text {non-dominant }}}^{\left(\frac{f_{3 d B}-f_{-3 d B}}{f_{3 d B} f_{-3 d B}}\right)})}{}\right.
$$

From Eq. 16 and 9, it can be seen that, as compared to the non-degenerated transconductor, the noise power in degenerated transconductor is a factor $I_{\text {bias }}^{D G} / I_{\text {bias }}$ times higher. This excess noise is in addition to a non-dominant noise contributed by the switched capacitor based resistor. This higher noise trades-off with increased linearity, as degeneration transforms the transconductance from a $v_{i n}$ dependent non-linear value of $g m_{i}$ to an independent linear value of $f_{s} C_{R}$. Further, as highlighted previously, implementing the degenerating resistor as a switched capacitor allows integrating large resistors for degeneration necessary in the audio frequency range. 


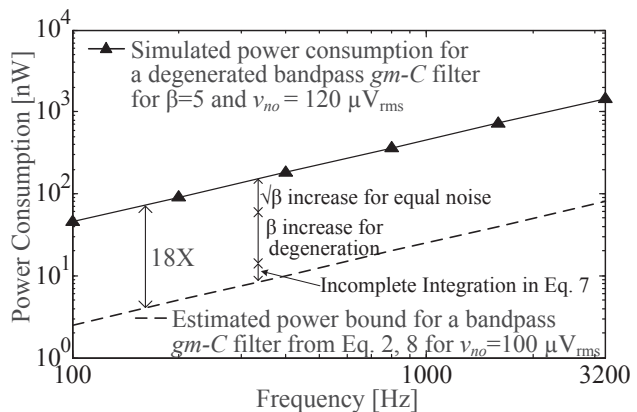

Fig. 5. Comparison of the simulated power consumption of the switchedcapacitor degenerated filter in $90 \mathrm{~nm}$ CMOS with the theoretical bounds on $g m-C$ filter as computed from Eq. 9 for constant integrated output noise

\section{Filter-BANK IMPLEMENTATION AND Results}

This section first details the design procedure for a single $\mathrm{BPF}$ and then presents the complete implementation of a 6 channel filter-bank by scaling the $f_{c}$. Table I highlights the targeted specifications for the filter-bank to be implemented in a $90 \mathrm{~nm}$ CMOS technology.

The $g m_{e f f}$ and the minimum $C_{l}$ necessary to meet the $f_{c}$ and the noise specifications for the first filter $\left(f_{c}=100 \mathrm{~Hz}\right)$ are estimated from Eq. 13 and 16 to be $g m_{e f f} \approx 25 n S$ and $C_{l} \approx$ $40 p F$ for a degeneration factor of $\beta=g m_{i} / f_{s} C_{R}=5$. For a Q-factor of $1, C_{l}=C_{i n}$ from Eq. 13. The capacitor values can be further reduced by maximizing the $g m / I D$ for $M_{i}$ and minimizing for $M_{t}, M_{p b}, M_{n b}$ respectively. The final values that achieve the required noise and linearity specifications are $C_{l}=25 p F$ and $g m_{e f f}=16 n S$. Though, the implementation here is for a Q-factor of 1, non unity Q-factors may be realized by varying the ratio $C_{i n} / C_{l}$ and/or the number of parallel $g_{m}$ cells in the forward $(N)$ and the feedback $(M)$ path.

The $f_{s}$ for switched-capacitor degeneration is chosen to be $512 \mathrm{kHz}$, which is greater than 100 times the maximum $f_{c}$, as this allows the intermodulation distortion to be attenuated by more than $40 \mathrm{~dB}$ under first order filter-roll off. Moreover, selecting such high $f_{s}$ allows to use a small $C_{R}=20 \mathrm{fF}$, thus minimizing the area impact.

To use the proposed filters in a complete filter-bank, $g m_{e f f}$ is scaled linearly from filter to filter by increasing the number of parallel cells in the forward $(N)$ and the feedback path $(M)$, which scales $f_{c} \propto \sqrt{M N}$. This allows for a constant integrated output noise independent of $f_{c}$. The periodic-ac and the periodic-noise analysis are used to simulate the magnitude response and the output noise voltage spectral density. These results for the first and the last channel of the bandpass filterbank are shown in Fig. 4. Results of the transient analysis for the maximum linear swing along with the integrated output noise for the first and the last filter are shown in Table II.

Figure. 5 compares the simulated power consumption of the switched-capacitor degenerated $g m-C$ BPF filters with the theoretical limit of regular $g m-C$ bandpass filters as a function of $f_{c}$ for a fixed integrated output noise. It can be seen from Fig. 5 that the power consumption is $\sim 18 X$ larger than the theoretical limit of non-degenerated $g m-C$ topologies. As illustrated in Fig. 5, this can be accounted for by the power
TABLE III

COMPARISON WITH STATE OF THE ART

\begin{tabular}{|c|c|c|c|c|c|}
\hline Specs. & This work & [3] & [4] & [5] & [1] \\
\hline Tech. & $90 \mathrm{~nm}$ & $0.8 \mu \mathrm{m}$ & $0.35 \mu \mathrm{m}$ & $\begin{array}{c}1.5 \mu \mathrm{m} \\
\mathrm{BiCMOS}\end{array}$ & $0.35 \mu \mathrm{m}$ \\
\hline$V_{D D}(\mathrm{~V})$ & 1.2 & 1.25 & 1.2 & 2.8 & $3.3^{1}$ \\
\hline DR (dB) & $63 / 66$ & $>62$ & 62.9 & 66 & 61.5 \\
\hline (\%) THD & $<2 /<5$ & 1 & $<1.1$ & 5 & 0.84 \\
\hline Power $(\mu W)$ & $0.09 / 0.9$ & 2.5 & $<16$ & $0.12 / 3.36$ & $0.19 / 2.85$ \\
\hline @ freq. (Hz) & $200 / 2 \mathrm{k}$ & $2 \mathrm{k}$ & $100 / 20 \mathrm{k}$ & $200 / 10 \mathrm{k}$ & $200 / 2 k$ \\
\hline $\begin{array}{c}\text { FoM } / 1 p \\
\frac{\text { Power } V_{D D}}{n f_{c} D R}\end{array}$ & $\begin{array}{c}0.19 / 0.14 \\
<2 /<5 \\
\text { thd }\end{array}$ & $<0.62$ & $<0.34$ & 0.23 & 1.3 \\
\hline
\end{tabular}

${ }^{1}$ assumed for a $0.35 \mu$ CMOS process

penalty incurred due to degeneration $(\beta X)$, maintaining the same integrated noise as the non-degenerated transconductor $(\sqrt{\beta} X)$ and the incomplete integration of noise in Eq. 7.

Table. III compares the degenerated $g m-C$ filter-bank to the state-of-the-art (SoTA) filter-banks for similar frequency range. It can be seen that the power consumption of this filter-bank compares favorably with the SoTA for a similar dynamic range inspite of the implementation in a sub-100nm technology. This affords a greater than $1.5 X$ improvement in the Figure-of-Merit (FoM) proposed in [6]. Also, this design, unlike the SoTA does not need non-standard CMOS transistors such as floating gate devices or BiCMOS technology.

\section{CONCLUSION}

This work evaluates the noise and frequency dependent power bounds on 2 OTA based $g m-C$ bandpass filters and proposes a novel alternative switched-capacitor based scalable degeneration technique. The introduced topology allows increasing the linear signal swing at the filter output to $484 m V_{p p},(D R=63 d B)$ and $700 m V_{p p},(D R=66 d B)$ for $<2 \%$ and $<5 \%$ THD respectively for a power supply voltage of $1.2 \mathrm{~V}$ in $90 \mathrm{~nm}$ CMOS. The power consumption of the proposed filter-bank compares favorably to SoTA and enhances the FoM by more than $1.5 \mathrm{X}$.

\section{ACKNOWLEDGMENT}

The authors thank Dr Filip Tavernier for useful discussions.

\section{REFERENCES}

[1] B. Rumberg and D. Graham, "A low-power and high-precision programmable analog filter bank," Circuits and Systems II: Express Briefs, IEEE Transactions on, vol. 59, no. 4, pp. 234-238, April 2012.

[2] K. Badami, S. Lauwereins, W. Meert, and M. Verhelst, " 24.2 contextaware hierarchical information-sensing in a $6 \mu \mathrm{w} 90 \mathrm{~nm}$ cmos voice activity detector," in Solid- State Circuits Conference - (ISSCC), 2015 IEEE International, Feb 2015, pp. 1-3.

[3] E. Rodriguez-Villegas, A. Yufera, and A. Rueda, "A 1.25-v micropower gm-c filter based on fgmos transistors operating in weak inversion," SolidState Circuits, IEEE Journal of, vol. 39, no. 1, pp. 100-111, Jan 2004.

[4] L. Pylarinos and K. Phang, "Low-voltage programmable gm-c filter for hearing aids using dynamic gate biasing," in Circuits and Systems, 2005. IEEE International Symposium on, May 2005, pp. 1984-1987 Vol. 3.

[5] C. Salthouse and R. Sarpeshkar, "A practical micropower programmable bandpass filter for use in bionic ears," Solid-State Circuits, IEEE Journal of, vol. 38, no. 1, pp. 63-70, Jan 2003.

[6] P. Corbishley and E. Rodriguez-Villegas, "A nanopower bandpass filter for detection of an acoustic signal in a wearable breathing detector," Biomedical Circuits and Systems, IEEE Transactions on, vol. 1, no. 3, pp. 163-171, Sept 2007. 